\title{
Tuning Fano Resonances with Graphene
}

Emani, Naresh K.; Chung, Ting-Fung; Prokopeva, Ludmila; Kildishev, Alexander; Chen, Yong P.; Boltasseva, Alexandra

\section{Published in:}

Proceedings of 2013 Conference on Lasers and Electro-Optics (CLEO)

Link to article, DOI:

10.1364/CLEO_SI.2013.CW3O.4

Publication date:

2013

Document Version

Publisher's PDF, also known as Version of record

Link back to DTU Orbit

Citation $(A P A)$ :

Emani, N. K., Chung, T-F., Prokopeva, L., Kildishev, A., Chen, Y. P., \& Boltasseva, A. (2013). Tuning Fano Resonances with Graphene. In Proceedings of 2013 Conference on Lasers and Electro-Optics (CLEO) [CW3O.4] IEEE. https://doi.org/10.1364/CLEO_SI.2013.CW3O.4

\section{General rights}

Copyright and moral rights for the publications made accessible in the public portal are retained by the authors and/or other copyright owners and it is a condition of accessing publications that users recognise and abide by the legal requirements associated with these rights.

- Users may download and print one copy of any publication from the public portal for the purpose of private study or research.

- You may not further distribute the material or use it for any profit-making activity or commercial gain

- You may freely distribute the URL identifying the publication in the public portal 


\title{
Tuning Fano Resonances with Graphene
}

\author{
Naresh K. Emani ${ }^{1}$, Ting-Fung Chung ${ }^{2}$, Ludmila Prokopeva ${ }^{1}$, Alexander Kildishev ${ }^{1}$, Yong P. Chen ${ }^{2}$, \\ and Alexandra Boltasseva ${ }^{*, 1,3}$ \\ ${ }^{1}$ School of Electrical and Computer Engineering and Birck Nanotechnology Center, Purdue University, West Lafayette, IN 47907, USA \\ ${ }^{2}$ Department of Physics and Birck Nanotechnology Center, Purdue University, West Lafayette, IN 47907, USA \\ ${ }^{3}$ DTU Fotonik, Department of Photonics Engineering, Technical University of Denmark, Lyngby, DK-2800, Denmark \\ Email: aeb@purdue.edu*
}

\begin{abstract}
We demonstrate strong electrical control of plasmonic Fano resonances in dolmen structures using tunable interband transitions in graphene. Such graphene-plasmonic hybrid devices can have applications in light modulation and sensing.

OCIS codes: (250.5403) Plasmonics; (160.4670) Optical materials; (160.3918) Metamaterials
\end{abstract}

\section{Introduction}

Graphene plasmonics offers an exciting possibility of combining conventional plasmonics and remarkable electrical and optical properties of graphene[1]. This can help in the realization of a variety of active plasmonic devices. The surface plasmon polariton wavelength in graphene has been shown to be 40-60 times smaller than free space wavelength [2]. Plasmonic nanoantennas were also shown to enhance photosensitivity of graphene [3]. In this direction, we recently demonstrated a graphene-antenna hybrid device for electrically controlling the damping of plasmonic resonance [4]. We used the gate controlled interband transitions to modulate the width of the plasmonic resonance (see also [ $[5])$. In this paper we further extend this approach to identify structures that would show enhanced tunability.

\section{Numerical Approach and Validation}

To design useful devices with graphene it is essential to develop accurate models for numerical simulations. Graphene is vanishingly thin monolayer of $\mathrm{sp}^{2}$ carbon atoms which poses unique challenges for numerical treatment. In numerical simulations, we can treat graphene as an effective medium with a thickness of $1 \mathrm{~nm}$. We believe an effective thickness of $1 \mathrm{~nm}$ is reasonable considering the inhomogeneties in CVD-grown graphene, which can lead to a graphene layer that is thicker than the ideal thickness. We obtain the dielectric function, which is commonly used in numerical modeling of optical processes, from the 2D sheet conductivity, $\sigma(\omega)$ derived within the local random-phase approximation []], as shown below:

$$
\varepsilon_{r}(\omega)=\frac{i \sigma(\omega)}{\omega \varepsilon_{0} t_{g}}
$$

where $t_{g}$ is effective thickness of graphene sheet, $\omega$ is frequency of light and $\varepsilon_{0}$ is the permittivity of vacuum. This approach was validated by comparing with gate-dependent optical transmission measurements which are shown in Fig. 1(a). The incident photon is most sensitive to carrier concentration in graphene when its energy is around $2 \mathrm{E}_{\mathrm{F}}$. Therefore, we see a matching peak in the normalized transmission spectrum. When the gate voltage is increased then the $\mathrm{E}_{\mathrm{F}}$ moves to higher energies or lower wavelengths. A gate voltage of $-30 \mathrm{~V}$ results in $2 \mathrm{E}_{\mathrm{F}}$ peak at $0.5 \mathrm{eV}$, which corresponds to a carrier density of $4.6 \times 10^{12} \mathrm{~cm}^{-2}$. At low gate voltages we expect the carrier concentration to be below $1 \times 10^{12} \mathrm{~cm}^{-2}$. The full wave simulations performed using Spatial Harmonic Analysis (SHA) [7] method are shown in Fig. 1(b). We see good agreement with the experimental trends except for the discrepancies at longer wavelengths.

\section{Tunable Fano Resonances}

The tunability of a resonance depends strongly on the strength of interaction between graphene and nanoantennas. After proof of principle demonstration with bowtie antennas [4], we investigated the tunability of Fano resonances in dolmen structure (shown in Fig. 2(a)) []]. In the dolmen structure overlap of dipolar and quadrupolar modes leads to a Fano resonance, and hence we expect large enhancement associated with a dark mode. With careful selection of geometrical parameters we identified structures resonating between 2.5-3.5 $\mu \mathrm{m}$. The results of numerical simulations are shown in Fig. 2(b) and 2(c). We note that the stronger enhancement in dolmen structures results in 
much stronger impact on plasmonic resonance. In this contribution we will also discuss experimental demonstrations of this enhanced tunability using the simulated designs, which is currently in progress.
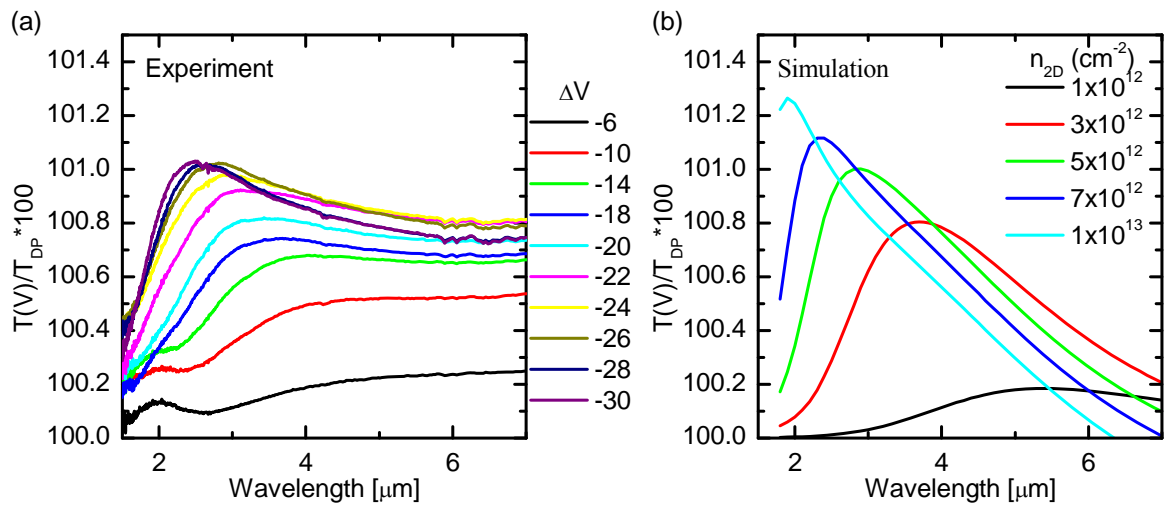

Fig 1. a) Experimentally measured relative change in optical transmission of graphene with gate voltage; b) SHA simulations of relative transmission with graphene sheet carrier density as a parameter.
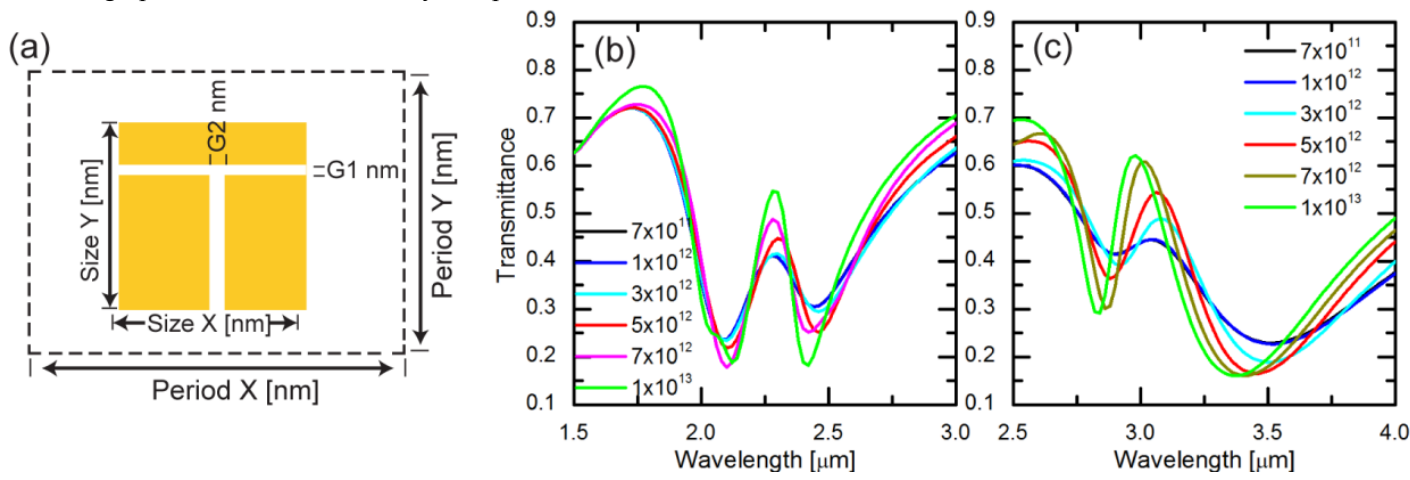

Fig 2. a) Schematic depicting the sample geometry used in numerical simulations; (b)-(c) Change in amplitude of the resonance as the carrier concentration is varied from $7 \times 10^{11}-1 \times 10^{13} \mathrm{~cm}^{-2}$.

Acknowledgements: This work was supported in part by ARO MURI grant 56154-PH-MUR (W911NF-09-10539), NSF MRSEC grant DMR-1120923.

\section{References}

1. A. Grigorenko, M. Polini, and K. Novoselov, "Graphene plasmonics," Nature Photonics 6, 749-758 (2012).

2. J. Chen, M. Badioli, P. Alonso-Gonzalez, S. Thongrattanasiri, F. Huth, J. Osmond, M. Spasenovic, A. Centeno, A. Pesquera, P. Godignon, A. Zurutuza Elorza, N. Camara, F. Javier Garcia de Abajo, R. Hillenbrand, and F. H. L. Koppens, "Optical nano-imaging of gatetunable graphene plasmons," Nature 487, 77-81 (2012).

3. Z. Fang, Z. Liu, Y. Wang, P. M. Ajayan, P. Nordlander, and N. J. Halas, "Graphene-Antenna Sandwich Photodetector," Nano Letters 12, 3808-3813 (2012).

4. N. K. Emani, T.-F. Chung, X. Ni, A. V. Kildishev, Y. P. Chen, and A. Boltasseva, "Electrically Tunable Damping of Plasmonic Resonances with Graphene," Nano Letters 12, 5202-5206 (2012).

5. J. Kim, H. Son, D. J. Cho, B. Geng, W. Regan, S. Shi, K. Kim, A. Zettl, Y. R. Shen, and F. Wang, "Electrical Control of Optical Plasmon Resonance with Graphene," Nano Letters 12, 5598-5602 (2012).

6. $\quad$ F. H. L. Koppens, D. E. Chang, and F. J. García de Abajo, "Graphene Plasmonics: A Platform for Strong Light-Matter Interactions," Nano Letters 11, 3370-3377 (2011).

7. X. Ni, Z. Liu, A. Boltasseva, and A. V. Kildishev, "The validation of the parallel three-dimensional solver for analysis of optical plasmonic bi-periodic multilayer nanostructures," Applied Physics A: Materials Science \& Processing 100, 365-374 (2010).

8. N. Verellen, Y. Sonnefraud, H. Sobhani, F. Hao, V. V. Moshchalkov, P. V. Dorpe, P. Nordlander, and S. A. Maier, "Fano resonances in individual coherent plasmonic nanocavities," Nano Letters 9, 1663-1667 (2009). 